\title{
FINITE INVERSE PERFECT SEMIGROUPS AND THEIR CONGRUENCES
}

\author{
HOWARD HAMILTON and TAKAYUKI TAMURA
}

(Received 18 June 1980; revised 2 February 1981)

Communicated by T. E. Hall

\begin{abstract}
In this paper we characterize the structure of finite inverse perfect semigroups and study congruences on those semigroups, in particular we study those semigroups that have modular lattice of congruences.
\end{abstract}

1980 Mathematics subject classification (Amer. Math. Soc): 20 M 10.

\section{Introduction}

Let $S$ be a semigroup and $\rho$ a congruence on $S$. If $a \in S, a \rho$ denotes the $\rho$-class containing $a$. Following Vagner (1968), a congruence $\rho$ is called perfect if $(a \rho)(b \rho)=(a b) \rho$ for all $a, b \in S$, as sets. A semigroup $S$ is called perfect if all congruences on $S$ are perfect. Groups are familiar examples of perfect semigroups. Perfect semigroups were studied by Fortunatov $(1970,1972,1974,1977)$. In this paper we completely characterize the structure of finite inverse perfect semigroups and study congruences on those semigroups, especially we study those that have modular lattice of congruences. In Section 1 we determine the structure of finite inverse perfect semigroups. There are two types: one type is a chain of groups, the other type is a chain of groups with a Brandt semigroup as an ideal component. The results for type 1 are due to Fortunatov (1972). In Section 2 we consider their construction and the isomorphism conditions. We discuss how to obtain their congruences in Section 3. Unlike groups, finite inverse semigroups do not all have modular lattices of congruences even if they

( Copyright Australian Mathematical Society 1982 
are perfect. In Section 4 we completely determine finite inverse perfect semigroups with modular lattice of congruences. For preliminary knowledge about inverse semigroups, completely simple semigroups, ideal extensions, semilattice of groups, the reader is referred to Clifford and Preston (1961) and Petrich (1973).

\section{Structure}

LEMMA 1.1. Let $S=\cup_{i \in \Gamma} S_{i}$ be the greatest semilattice decomposition of $S$. If $S$ is an inverse semigroup, each $S_{i}$ is a semilattice-indecomposable inverse semigroup.

Proof. By Tamura (1956) or Petrich (1973), each $S_{i}$ is semilattice-indecomposable. We can easily see $S_{i}$ is an inverse semigroup.

Lemma 1.2. (Fortunatov (1972)) Perfectness is preserved under homomorphic images.

LemMa 1.3. (Fortunatov (1972)) A (lower) semilattice $S$ is perfect if and only if it is a chain.

LeMma 1.4. Let $S$ be a perfect semigroup. If $I$ is an ideal of $S$ and $I \neq S$, $I \neq\{0\}$, then $I$ is a completely prime ideal of $S$.

Proof. Suppose $x, y \in S \backslash I$ and $x y \in I$. Since $\{x\}\{y\} \neq I, S$ is not perfect. Hence $S \backslash I$ is a subsemigroup of $I$.

Let $S$ be a finite inverse semigroup and $S=\cup_{i \in \Gamma} S_{i}$ be the greatest semilattice decomposition of $S$. Assume $S$ is perfect. By Lemmas 1.2 and 1.3, or by Fortunatov (1972), $\Gamma$ is a chain, say $\Gamma=\{0,1, \ldots, n\}$ with the usual ordering.

LEMma 1.5. $S_{i}$ is a group for all $i \in \Gamma, i \neq 0$, and $S_{0}$ is either a group or $a$ Brandt semigroup.

Proof. Suppose $S_{i}, i>0$, is not simple, let $J \neq S_{i}$ be an ideal of $S_{i}$ (namely, $\left.1 \leqslant|J|<\left|S_{i}\right|\right)$. Then $\bar{I}=\cup_{j<i} S_{j} \cup S_{i} J S_{i}$ is an ideal of $S$, and $\bar{I} \neq S$. By Lemma $1.4 \bar{I}$ is a completely prime ideal of $S$, hence $J$ is a completely prime ideal of $S_{i}$. This contradicts semilattice-indecomposability of $S_{i}$. Thus $S_{i}$ is completely simple because of finiteness. Since $S_{i}$ is an inverse semigroup, $S_{i}$ is a 
group for all $i \in \Gamma, i \neq 0$. In case $S$ has no zero, let $K$ be the minimal ideal of $S$. In case $S$ has zero, let $K$ be a 0 -minimal ideal of $S$ with $K \cap S_{0} \neq \varnothing$. In both cases $K \subseteq S_{0}$. Suppose $K \neq S_{0}$. By the same argument as $\bar{I}$ above, $K$ is a completely prime ideal of $S_{0}$, a contradiction. Hence $K=S_{0}$. Since $S_{0}$ is a completely $[0-]$ simple inverse semigroup, we conclude that $S_{0}$ is either a group or a Brandt semigroup.

In this paper a Brandt semigroup whose ground group is $G$ and whose sandwich matrix is the $m \times m$ identity matrix is denoted by $\mathscr{K}^{0}(m, G)$. Since $S_{0}$ is semilattice-indecomposable, we adopt the convention that a Brandt semigroup $\mathscr{T}^{0}(m, G)$ satisfies $m>1$. The universal relation and the equality relation are respectively denoted by

$$
\omega(X)=X \times X, \quad \iota(X)=\{(x, x): x \in X\} .
$$

Let $\Omega_{r}\left(S_{j}\right)$ denote the semigroup of right translations of $S_{j}$ which are linked with some left translations of $S_{j}$. The system of homomorphisms used in the definition of the binary operation on $\bigcup_{i=0}^{n} S_{i}$ are given by

$$
\begin{array}{rll}
i>j & \varphi_{i j}: S_{i} \rightarrow \Omega_{r}\left(S_{j}\right), & p \rightarrow p \varphi_{i j}, \quad p \in S_{i} \\
& p \varphi_{i j}: S_{j} \rightarrow S_{j}, \quad x \rightarrow x\left(p \varphi_{i j}\right), \quad x \in S_{j}
\end{array}
$$

with a dual action for $\psi_{i j} \in \Omega_{l}\left(S_{j}\right)$, the semigroup of left translations of $S_{j}$. If $x \in S_{i}$ and $y \in S_{j}$ for $i \geqslant j$ then $y x=y\left(x \varphi_{i j}\right)$ and $x y=\left(\psi_{i j} x\right) y$. In case $i, j>0$, $S_{i}$ and $S_{j}$ are groups and $p \varphi_{i j}$ is interpreted as an element of $S_{j}$ since $p \varphi_{i j}$ is an inner right translation of $S_{j}$, and hence $\varphi_{i j}: S_{i} \rightarrow S_{j}$ is a homomorphism of $S_{i}$ into $S_{j}$. For $\cup_{i=1}^{n} S_{i}$ the set $\left\{\varphi_{i j}: i>j\right\}$ is the so-called transitive system, namely, it satisfies

(i) $\varphi_{i i}$ is the identical map of $S_{i}$,

(ii) $\varphi_{i j} \varphi_{j k}=\varphi_{i k}, i \geqslant j \geqslant k$.

However condition (ii) will be extended to $S_{0}$ even if $S_{0}$ is not a group (see Lemma 2.2). Since $S_{i}(i=0,1, \ldots)$ is left reductive, for $p \in S_{i}$ and $x \in S_{j}$, there is a unique $z \in S_{i}$ such that

$$
\left(p \varphi_{i j}\right)\left(x \varphi_{j j}\right)=z \varphi_{j j} \text { and } z=\left(\psi_{i j} p\right) x .
$$

Thus $\psi_{i j}$ is determined by $\varphi_{i j}$.

LEMMA 1.6. $S=\cup_{i=0}^{n} S_{i}$ is composed by permutations.

Proof. $S_{0}$ is an ideal of $S$. By perfectness, $S_{0} p=S_{0}$ hence $p \varphi_{i 0}$ is a permutation on $S_{0}$ for all $p \in S_{i}$ for all $i \neq 0$, since $S_{0}$ is finite. Obviously $p \varphi_{i j}$ is a permutation for all $p \in S_{i}$, all $i>j>0$. 
Lemma 1.7. (Fortunatov (1972)) For all $i>j>0$ (for all $i>j>0$ if $S_{0}$ is $a$ group), $\varphi_{i j}$ is surjective.

$S$ is called type 1 if $S_{0}$ is a group; type 2 if $S_{0}$ is a Brandt semigroup.

We want to prove that if $S=\cup_{i \in \Gamma} S_{i}$ satisfies Lemmas $1.5,1.6$ and 1.7 then $S$ is perfect. This has been proved for type 1 semigroups by Fortunatov (1972), so we need only prove it for type 2 semigroups.

Let $S_{0}=\Re^{0}(m, G)=\{0\} \cup\{(k, x, l): x \in G, k, l=1, \ldots, m\}$ where $G$ is a finite group and $\left(k_{1}, x, l_{1}\right)\left(k_{2}, y, l_{2}\right)=\left(k_{1}, x y, l_{2}\right)$ if $l_{1}=k_{2} ; 0$ if $l_{1} \neq k_{2}$. Let $\rho$ be a congruence on $S$.

Lemma 1.8. Assume $i>0$ and $a \rho b$ for some $a \in S_{0}, b \in S_{i}$. Then

$$
\omega\left(\bigcup_{l<i} S_{l}\right) \subseteq \rho
$$

Proof. For all $x \in S_{0}, x\left(a \varphi_{00}\right) \rho x\left(b \varphi_{i 0}\right)$. By Lemma $1.6 b \varphi_{i 0}$ is a permutation on $S_{0}$. There is $z \in S_{0}, z \neq 0$, such that $z a=0$ but $z b \neq 0$. It follows that $x \rho b$ for all $x \in S_{0}$. On the other hand $I=\left\{c \in S: x \rho c\right.$ for all $\left.x \in S_{0}\right\}$ is an ideal of $S$ and $I \cap S_{i} \neq \varnothing$. Since $S_{i}$ is a group, $\cup_{l<i} S_{l} \subseteq I$.

Lemma 1.9. (Clifford and Preston (1967), Tamura (1960)). Let $\rho_{0}=\rho \mid S_{0}$. If $\rho_{0} \neq \omega\left(S_{0}\right)$ then $\rho_{0}$ is determined by a group-congruence $\tau$ on $G$ such that $0 \rho_{0} 0$ and $\left(k_{1}, x, l_{1}\right) \rho_{0}\left(k_{2}, y, l_{2}\right)$ if and only if $k_{1}=k_{2}, x \tau y, l_{1}=l_{2}$.

We denote $\rho_{0}$ by $\rho_{0}(\tau)$.

Lemma 1.10. If $b \in S_{i}, a \in S_{j}, x \in S_{k}$ and if $x \rho a b$ then $x \in(a \rho)(b \rho)$.

Proof. If $i, j, k \neq 0$, the argument for Type 1 is available. By Lemmas 1.8 and 1.9 we need consider only the folowing cases:

(I) $a \rho=b \rho=\cup_{l<k} S_{l}$ where $k=\max (i, j)$;

(II) $a \rho=\cup_{l<j} S_{l}$ and $b \rho \cap S_{0}=\varnothing ;$ (II') $a \rho \cap S_{0}=\varnothing$ and $b \rho=$ $\cup_{l \leq i} S_{l}$;

(III) $a \rho \subseteq S_{0}$ and $b \rho \cap S_{0}=\varnothing$; (III') $a \rho \cap S_{0}=\varnothing$ and $b \rho \subseteq S_{0}$;

(IV) $a \rho \subseteq S_{0}$ and $b \rho \subseteq S_{0}$.

For (I), perfectness follows from the fact that $a b \in \cup_{l<k} S_{l}$ and $S_{l}^{2}=S_{l}$ for all $l$. For (II), necessarily $i>j$; $a \rho$ is an ideal of $S$. Let $y \in b \rho$. By Lemma 1.6, $y$ acts on $S l$ by permutation for all $l \leqslant j$, and hence acts on $a \rho$ by permutation. 
Then we have

$$
(a b) \rho \subseteq a \rho=(a \rho) y \subseteq(a \rho)(b \rho) .
$$

For (III), let $y \in b \rho$. Certainly $(a \rho) y \subseteq(a b) \rho$, but since $y$ acts on $S_{0}$ by permutation, we see that $(a \rho) y=(a b) \rho$ for all $y \in b \rho$ by Lemma 1.9. Hence $(a \rho)(b \rho)=(a b) \rho$. Case (IV) follows since completely 0-simple semigroups are perfect by Fortunatov (1972).

Summarizing Lemmas 1.5 to 1.10 we have

THEOREM 1.11. Let $S$ be a finite inverse semigroup and $S=\cup_{i \in \Gamma} S_{i}$ be the greatest semilattice decomposition of $S$ where $\Gamma$ denotes a lower semilattice. Then $S$ is perfect if and only if

(1.11.1) $\Gamma$ is a chain: $\Gamma=\{0,1, \ldots, n\}$ with operation $i \cdot j=\min \{i, j\}$,

(1.11.2) $S_{0}$ is either a group or a Brandt semigroup and $S_{i}$ is a group for all $i>0$,

(1.11.3) $S$ is composed by permutations,

(1.11.4) for all $i>j>0$ (for all $i>j>0$ if $S_{0}$ is a group), $\varphi_{i j}: S_{i} \rightarrow S_{j}$ is surjective.

\section{Construction and isomorphism criterion}

A type 1 semigroup $S$ is determined by a transitive system of surjective homomorphisms $\left\{\varphi_{i j}\right\}$ of finite groups $S_{i}$ into $S_{j} ; S$ is denoted by

$$
S=\left(\bigcup_{i=1}^{n} S_{i}, \varphi_{i j}, i \geqslant j\right)
$$

Let another type 1 semigroup be

$$
S^{\prime}=\left(\bigcup_{i=1}^{n^{\prime}} S_{i}^{\prime}, \varphi_{i j}^{\prime}, i \geqslant j\right) .
$$

THEOREM 2.1. $S \cong S^{\prime}$ if and only if $n=n^{\prime}$ and for each $i$ there is an isomorphism $h_{i}$ of $S_{i}$ onto $S_{i}^{\prime}$ such that

$$
\varphi_{i j} h_{j}=h_{i} \varphi_{i j}^{\prime} \quad \text { for } i \geqslant j .
$$

LEMMA 2.2. Let $S=\cup_{i=0}^{n} S_{i}$ be of type 2 .

(2.2.1) Let $i>j>0$ and $k>j>0$, and $p \in S_{i}, q \in S_{k}$. If $p \varphi_{i j}=q \varphi_{k j}$ then $p \varphi_{i 0}=q \varphi_{k 0}$.

(2.2.2) $\varphi_{i j} \varphi_{j 0}=\varphi_{i 0}$ for $i>j>0$. 
Proof of (2.2.1). The map $p \mapsto p \varphi_{i 0}$ induces a homomorphism of $\cup_{i>1} S_{i}$ into the group of permutations of $S_{0}$. Now $p \varphi_{i j}=q \varphi_{k j}$ implies $x p=x q$ for all $x \in S_{j}$; then $\left(x \varphi_{j 0}\right)\left(p \varphi_{i 0}\right)=\left(x \varphi_{j 0}\right)\left(q \varphi_{k 0}\right)$. Since $x \varphi_{j 0}$ is a permutation of $S_{0}$, we have $p \varphi_{i 0}=q \varphi_{k 0}$.

Proof of (2.2.2). Let $p \varphi_{i j}=r \varphi_{i j}, r \in S_{j}$. By (2.2.1) $p \varphi_{i 0}=r \varphi_{j 0}$. For $z \in S_{0}$, $z\left(p \varphi_{i 0}\right)=z\left(r \varphi_{j 0}\right)=z\left(p \varphi_{i j}\right) \varphi_{j 0}$, hence $p \varphi_{i 0}=p \varphi_{i j} \varphi_{j 0}$ for all $p \in S_{i}$.

The aim now is to construct type 2 semigroups from type 1 semigroups and Brandt semigroups. Let $F_{M}$ be a permutation group over $M=\{1,2, \ldots, m\}, G$ a finite group and $\mathcal{G}_{M}(G)$ the group of mappings of $M$ into $G$ with operation * defined by

$$
j\left(g_{1} * g_{2}\right)=\left(j g_{1}\right)\left(j g_{2}\right), \quad g_{1}, g_{2} \in \mathcal{G}_{M}(G), j \in M .
$$

Let $\left(\cup_{i=1}^{n} S_{i}, \varphi_{i j}, i \geqslant j\right)$ be of type 1 . For $S_{1}$, choose $M=\{1, \ldots, m\}, F_{M}$ and a homomorphism $f$ of $S_{1}$ onto $F_{M}$. This is well known as a representation of $S_{1}$ by permutations (for example see Wielandt (1964)). Of course the representation $S_{1} \rightarrow F_{M}$ is not required to be transitive. Given $F_{M}$ and $f$, we can choose a finite group $G$ and a mapping $g$ of $S_{1}$ onto $\mathcal{G}_{M}(G)$ such that

$$
(a g) *((a f)(b g))=(a b) g \text { for all } a, b \in S_{1} \text {. }
$$

This is always possible since the trivial group can be chosen as $G$. We do not consider the determination of all $G, \mathscr{S}_{M}(G)$ and $g$ satisfying (2.3), but note that if $e$ is the identity element of $S_{1}$ then $e g$ is necessarily the map which carries all elements of $M$ to the identity element of $G$.

Given $F_{M}, G, f$ and $g$, define a Brandt semigroup $S_{0}=\mathscr{N}^{0}(m, G)$, where $m=|M|$, and define $\varphi_{10}$ by

$$
\varphi_{10}: S_{1} \rightarrow \Omega_{r}\left(S_{0}\right), \quad a \varphi_{10}=(a g, a f),
$$

where $(a g, a f): S_{0} \rightarrow S_{0}$ is defined by

$$
(i, x, j) \mapsto(i, x \cdot(j(a g)), j(a f)) .
$$

By (2.3) we see that $\varphi_{10}$ is a homomorphism of $S_{1}$ into $\Omega_{r}\left(S_{0}\right)$. We denote $\varphi_{10}$ by $\varphi_{10}=(g, f)$.

By means of $\varphi_{10}$ we can construct a composition $S=\cup_{i=0}^{n} S_{i}$ of type 2 of $S_{0}$ and $\cup_{i-1}^{n} S_{i}$. All semigroups of type 2 can be obtained in this manner. $S$ is denoted by

$$
S=\left(\bigcup_{i=0}^{n} S_{i}, \varphi_{10}, \varphi_{i j}, i \geqslant j\right)
$$

or

$$
S=\left(\bar{S}_{1}, G, m, g, f\right), \quad S_{0}=\mathfrak{T}^{0}(m, G),
$$


where

$$
\bar{S}_{1}=\left(\bigcup_{i=1}^{n} S_{i}, \varphi_{i j}, i>j\right) \text {, and } \varphi_{10}=(g, f) \text {. }
$$

Let $S^{\prime}=\left(\bar{S}_{1}^{\prime}, G^{\prime}, m^{\prime}, g^{\prime}, f^{\prime}\right), \bar{S}_{1}^{\prime}=\left(\cup_{i=1}^{n} S_{i}^{\prime}, \varphi_{i j}^{\prime}, i>j\right), \varphi_{10}^{\prime}=\left(g^{\prime}, f^{\prime}\right)$.

THEOREM 2.4. $S \cong S^{\prime}$ if and only if the following conditions are satisfied:

(2.4.1) $\bar{S}_{1} \cong \bar{S}_{1}^{\prime}$

(2.4.2) $m=m^{\prime}$;

(2.4.3) there is an isomorphism $\gamma$ of $G$ onto $G^{\prime}$ and a permutation $\alpha$ of $M=\{1,2, \ldots, m\}$ such that for all $a \in S_{1}$

$$
(a f) \cdot \alpha=\alpha \cdot\left(a h_{1}\right) f^{\prime}
$$

and

$$
(a g) \cdot \gamma=\alpha \cdot\left(a h_{1}\right) g^{\prime}
$$

where $h_{1}: S_{1} \rightarrow S_{1}^{\prime}$ is defined in Theorem 2.1 .

PRoOF. First we have $S \cong S^{\prime}$ if and only if $\bar{S}_{1} \cong \bar{S}_{1}^{\prime}$ and there is an isomorphism $h_{0}: S_{0} \rightarrow S_{0}^{\prime}$ such that

$$
h_{0}^{-1}\left(a \varphi_{10}\right) h_{0}=\left(a h_{1}\right) \varphi_{10}^{\prime} \text { for all } a \in S_{1} .
$$

On the other hand $h_{0}$ is determined by a permutation $\alpha$ of $M$ and an isomorphism $\gamma$ of $G$ to $G^{\prime}$ such that

$$
(i, x, j) h_{0}=(i \alpha, x \gamma, j \alpha) \text {. }
$$

One can prove the theorem by using these.

\section{Congruences}

In this section we study congruences on finite inverse perfect semigroups.

LEMMA 3.1. (Birkhoff (1967)) A congruence on a finite chain can be obtained as a disjoint union of intervals. (Singleton intervals are admitted.)

If $\Gamma$ is a finite chain, namely, $\Gamma=\{1,2, \ldots, n\}$ with operation $i \cdot j=$ $\min \{i, j\}$, then each congruence $\sigma$ on $\Gamma$ is associated with a partition $\left\{I_{j}\right.$ : $1 \leqslant j \leqslant m\}$ where

$$
\begin{aligned}
I_{j} & =\left\{x \in \Gamma: i_{j} \leqslant x \leqslant i_{j+1}-1\right\}, \quad j=1, \ldots, m-1, \\
I_{m} & =\left\{x \in \Gamma: i_{m} \leqslant x \leqslant n\right\}
\end{aligned}
$$


and

$$
1=i_{1}<i_{2}<\cdots<i_{m}<n .
$$

The $i_{j}$ is called the initial of $I_{j}$, and the initial of the class $k \sigma$ containing $k$ is denoted by $\bar{k}$, that is, $\bar{k} \in k \sigma$ and $\bar{k}<x$ for all $x \in k \sigma$. The set of initials with respect to $\sigma$ is denoted by $\mathscr{G}_{\sigma}$.

The next result for a type 1 semigroup $S=\left(\cup_{i=1}^{n} S_{i}, \varphi_{i j}, i>j\right)$ specializes results of Reilly and Scheiblich (1967).

THEOREM 3.2. $\rho$ is an idempotent separating congruence on $S$ if and only if $\rho=\cup_{i=1}^{n} \rho_{i}$ where $\rho_{i}$ is a congruence on $S_{i}$ so that $x \rho_{i} y$ implies $x \varphi_{i j} \rho_{j} y \varphi_{i j}$ for all $i$ and $j, 0<j \leqslant i \leqslant n$ and $x, y \in S_{i}$.

Assume $\sigma$ is a congruence on $\Gamma$ and $\rho=\cup_{i \in \Gamma} \rho_{i}$ is an idempotent-separating congruence on $S$. For each $l \in g_{\sigma}$, define $\eta_{l}$ on $\bigcup_{i \in l_{\sigma}} S_{i}$ as follows: $x \eta_{l} y$ if and only if $x \in S_{i}, y \in S_{j}, i, j \in l \sigma, x \varphi_{i l} \rho_{l} y \varphi_{j l}$. Now define $\eta$ by $\eta=\cup_{l \in g_{0}} \eta_{l}$, denoted by $\eta=\left(\sigma ; \rho_{i}, i \in \Gamma\right)$.

THEOREM 3.3. The $\eta$ is a congruence on $S$. Every congruence on $S$ can be obtained in this manner.

Proof. The proof of compatibility is routine. To prove the second part, let $\rho$ be a congruence on $S$. Let $\rho_{i}=\rho \mid S_{i}$. Then $\cup \rho_{i}$ is a congruence on $S$. Define $\sigma$ on $\Gamma$ by $i \sigma j$ if and only if $x \rho y$ for some $x \in S_{i}$ and $y \in S_{j}$. Let $\eta=\left(\sigma ; \rho_{i}, i \in \Gamma\right)$. Assume $a \rho b$ where $a \in S_{i}, b \in S_{j}, i>j$. Let $l \in \mathscr{G}_{\text {o }}$ such that $i, j \in l \sigma$. Let $e_{l}$ denote the identity element of $S_{l}$. Then

$$
a \varphi_{i l}=e_{l}\left(a \varphi_{i l}\right)=e_{l} a \rho_{l} e_{l} b=e_{l}\left(b \varphi_{j l}\right)=b \varphi_{j l}
$$

hence $a \eta b$. Conversely assume $a \eta b, a \in S_{i}, b \in S_{j}, i, j \in l \sigma$. Since $i \sigma l, x \rho y$ for some $x \in S_{i}, y \in S_{l}$. Let $a=x u, u \in S_{i}$. Then $a \varphi_{i l}=e_{l}\left(a \varphi_{i l}\right)=e_{l} a \rho e_{\nu} y u=$ yupa. Similarly $b \varphi_{j l} \rho b$. Hence $a \rho b$ follows.

COROLlary 3.4. If $\rho_{i}=\imath\left(S_{i}\right)$ for each $i \in l \sigma$, then $\eta_{l}$ is the smallest group congruence on $\cup_{i \in l \sigma} S_{i}$. In particular $\eta=\left(\omega(\Gamma) ; \iota\left(S_{i}\right), i \in \Gamma\right)$ is the smallest group congruence on $S$, and $\eta \mid S_{1}=\iota\left(S_{1}\right)$ (see Howie (1976)).

Corollary 3.5. I is an ideal of $S=\left(\cup_{i \in \Gamma} S_{i}, \varphi_{i j}, i>j\right)$ if and only if there is $\xi=\left(\sigma ; p_{i}, i \in \Gamma\right)$ on $S$ such that $I=\cup_{i \in l \sigma} S_{i}$ and $\rho_{i}=\omega\left(S_{i}\right)$ for all $i \in 1 \sigma$ (or equivalently by Theorem 3.3, $\rho_{1}=\omega\left(S_{1}\right)$ ). 
The ideal $I$ is called the ideal due to $\xi$, denoted by $I(\xi)$.

Let $S=(\bar{S}, G, m, g, f), S_{0}=\mathscr{N}^{0}(m, G)$ where $\bar{S}=\left(\cup_{i \in \Gamma} S_{i}, \varphi_{i j}, i>j\right), \Gamma=$ $\{1,2, \ldots, n\}$.

Let $\xi$ be a congruence on $\bar{S}$, say $\xi=\left(\sigma ; \rho_{i}, i \in \Gamma\right)$. Let $\bar{S}_{\xi}^{*}=\bar{S} \backslash I(\xi)$ if $\rho_{1}=\omega\left(S_{1}\right)$. For any $\xi$, choose a congruence $\rho_{0}=\rho_{0}(\tau)$ on $S_{0}$ such that $a \rho_{1} b$, $a, b \in S_{\mathrm{l}}$, implies $a f=b f$ and $j(a g) \tau j(b g)$ for all $j \in M=\{1, \ldots, m\}$; equivalently,

$$
a \rho_{1} b \text { implies } z\left(a \varphi_{10}\right) \rho_{0} z\left(b \varphi_{10}\right) \text { for all } z \in S_{0} \text {. }
$$

Then we say a pair $\left(\rho_{0}, \rho_{1}\right)$ satisfies condition (3.6), or a pair $\left(\rho_{0}, \xi\right)$ satisfies condition (3.6).

THEOREM 3.7. For any congruence $\xi$ on $\bar{S}$ and a pair $\left(\rho_{0}, \xi\right)$ satisfying (3.6), define $\eta_{1}=\rho_{0} \cup \xi$. If $\xi=\left(\sigma ; \rho_{i}, i \in \Gamma\right)$ and $\rho_{1}=\omega\left(S_{1}\right)$, define $\eta_{2}$ by $\eta_{2}=$ $\omega\left(I(\xi) \cup S_{0}\right) \cup\left(\xi \mid \bar{S}_{\xi}^{*}\right)$. Then $\eta_{1}$ and $\eta_{2}$ are congruences on $S$. Every congruence on $S$ is of the form of $\eta_{1}$ or $\eta_{2}$. The expressions for $\eta_{1}$ and $\eta_{2}$ are unique.

$\eta_{1}$ is called a congruence of the first kind; $\eta_{2}$ is of the second kind.

PRoof. Let $\eta$ be a congruence on $S$ and $\xi=\eta \mid \bar{S}$. By Lemma 1.8, if $x \eta y$ for some $x \in S_{0}$ and $y \notin S_{0}$ then $\eta$ has the form $\eta_{2}$. Condition (3.6) follows from compatibility. Conversely it is obvious that $\eta_{2}$ is a congruence on $S$. If no element of $S_{0}$ is $\eta$-related to an element not in $S_{0}$ and if $\rho_{0}=\eta \mid S_{0} \neq \omega\left(S_{0}\right)$ then $\rho_{0}=\rho_{0}(\tau)$ by Lemma 1.9. Compatibility yields the condition (3.6) on $\rho_{0}$. Conversely let $\eta_{1}=\xi \cup \rho_{0}$. We need verify only compatibility. It is easy to see that $\rho_{0}$ is compatible with every right and left translation of $S_{0}$, hence $\rho_{0}$ is compatible with all elements $z \in S$. Assume $a \xi b, a \in S_{i}, b \in S_{j}, i, j \geqslant 1$. Let $c \in S_{1}$. Then $a \xi b$ implies $c a \xi c b$, that is, $c\left(a \varphi_{i 1}\right) \rho_{1} c\left(b \varphi_{j 1}\right)$ whence $a \varphi_{i 1} \rho_{1} b \varphi_{j 1}$ since $\rho_{1}$ is a group congruence. Let $z \in S_{0}$. By using (3.6) and (2.2.2),

$$
z a=z\left(a \varphi_{i 0}\right) \rho_{0} z\left(b \varphi_{j 0}\right)=z b \text { for all } z \in S_{0}
$$

whence $z a \rho_{0} z b$. Next we want to show $a z \rho_{0} b z$ for all $z \in S_{0}$. Recall $\psi_{i 0} a$ is a left translation linked with $a \varphi_{i 0}$. For $x \in S_{0}, x(a z)=x\left(\left(\psi_{i 0} a\right) z\right)=$ $\left(x\left(a \varphi_{i 0}\right)\right) z \rho_{0}\left(x\left(b \varphi_{j 0}\right)\right) z=x\left(\left(\psi_{j 0} b\right) z\right)=x(b z)$. Since by Lemma 1.6, $a z=0$ if and only if $b z=0$ we assume $a z \neq 0$ and $b z \neq 0$ for some $z$, so $x(a z) \neq 0$ and $x(b z) \neq 0$ for some $x$. Since $\rho_{0} \neq \omega\left(S_{0}\right)$, the elements $x, a z$ and $b z$ have the form $x=(k, u, l), a z=(l, v, s), b z=(l, w, s)$ by Lemma 1.9. Let $x^{\prime}=\left(l, u^{-1}, k\right)$. Then $x^{\prime} x(a z) \rho_{0} x^{\prime} x(b z)$ implies $a z \rho_{0} b z$ as desired.

RemarK. As seen in the proof of Theorem 3.7, it follows that $a \rho_{1} b$ implies $\left(\psi_{10} a\right) z \rho_{0}\left(\psi_{10} b\right) z$ for all $z \in S_{0}$. Hence only (3.6) is required. 
COROLLARY 3.8. If $\bar{S}$ is a group, all nonuniversal congruences on $S$ are of the first kind.

Proof. Suppose $a \eta b$ for some $a \in S_{0}$ and some $b \in \bar{S}$. By Lemma 1.8, $\omega\left(S_{0}\right) \subseteq \eta$. Since the set $\left\{b \in \bar{S}: x \eta b\right.$ for $\left.x \in S_{0}\right\}$ is an ideal of $\bar{S}$, it must coincide with $\bar{S}$, and hence $\eta=\omega(S)$.

Corollary 3.9. If $\left|S_{1}\right|=1$, any pair $\left(\rho_{0}, \xi\right)$ satisfies condition (3.6) for a congruence $\xi$ on $\bar{S}$ and a congruence $\rho_{0}$ on $S_{0}$.

\section{Modularity}

Let $L$ be a lattice with join $\vee$ and meet $\wedge$, and $L^{1}$ the lattice obtained by adjoining a new element 1 to $L$ such that 1 is the greatest element of $L^{1}$, and join and meet in $L$ are preserved.

LEMma 4.1. (Birkhoff (1967)) $L^{1}$ is modular if and only if $L$ is modular. Modularity in lattices is preserved under sublattices, homomorphic images nad direct products.

A semigroup $D$ is called modular if the lattice $L(D)$ of congruences on $D$ is a modular lattice. In this section we determine the structure of finite inverse perfect modular semigroups.

LEMMA 4.2. A homomorphic image of a modular semigroup is modular.

Let $D^{0}=D \cup\{0\}$ be $D$ with zero 0 adjoined.

Lemma 4.3. For any semigroup $D, D^{0}$ is modular if and only if $D$ is modular.

Proof. For each $\xi \in L(D)$, define a congruence $(\xi, 0)$ on $D^{0}$ by $(\xi, 0)=\{(0,0)\} \cup \xi$.

$L(D)$ is isomorphic to a sublattice of $L\left(D^{9}\right)$ under $\xi \mapsto(\xi, 0)$. Hence if $L\left(D^{9}\right)$ is modular, $L(D)$ is modular. If $\xi \in L(D)$ such that $D / \xi$ has zero 0 we let $I(\xi)$ denote the pre-image of 0 under $D \rightarrow D / \xi$, and define a congruence $(\xi, 1)$ on $D^{0}$ by

$$
(\xi, 1)=\omega(I(\xi) \cup\{0\}) \cup\left(\xi \mid D_{\xi}^{*}\right)
$$


where $D_{\xi}^{*}=D \backslash I(\xi)$. Every congruence on $D^{0}$ can be uniquely expressed as either $(\xi, 0)$ or $(\xi, 1)$ for $\xi \in L(D)$. Thus

$$
L\left(D^{0}\right)=\{(\xi, 0): \xi \in L(D)\} \cup\{(\xi, 1): \xi \in L(D), D / \xi \text { has zero }\} .
$$

Let $L(2)$ denote the lattice of two elements: $L(2)=\{0,1\}$. It can be shown that if $\xi, \eta \in L(D)$ and $i, j \in L(2)$, then

$$
\begin{aligned}
& (\xi, i) \cap(\eta, j)=(\xi \cap \eta, \min \{i, j\}), \\
& (\xi, i) \vee(\eta, j)=(\xi \vee \eta, \max \{i, j\}) .
\end{aligned}
$$

Thus $L\left(D^{9}\right)$ is isomorphic to a sublattice of the direct product $L(D) \times L(2)$. Hence if $L(D)$ is modular, $L\left(D^{9}\right)$ is modular.

LEMMA 4.4. Let $I$ be a completely prime ideal of a semigroup $D$ and $D \backslash I \neq \varnothing$. If $D$ is modular, then $D \backslash I$ is modular.

Proof. Let $\rho$ be a congruence on $D \backslash I$. For $\rho$, define a congruence $\rho^{0}$ on $D$ by $\rho^{0}=p \cup \omega(I)$. Then $L(D \backslash I)$ is isomorphic to a sublattice of $L(D)$ under $\rho \rightarrow \rho^{0}$. The conclusion follows.

Consider the following finite semigroups:

(M1) a chain;

(M2) a group;

(M3) a chain-group composition.

We explain (M3). Let $G$ be a group and let $G_{0}=G, G_{1}=G_{0} \cup\left\{0_{1}\right\}$ be $G_{0}$ with zero $0_{1}$ adjoined. By induction, let $G_{i}$ be $G_{i-1}$ with zero $0_{i}$ adjoined. Then $G_{n}$ is called a group with a chain $\Gamma=\{1, \ldots, n\}$ adjoined below, that is, the ideal extension of $\Gamma$ by $G^{0}$ by means of identity translations of $\Gamma$. We call it the identity-composition of a chain by a group or simply, a chain-group composition.

TheOREM 4.5. Assume $S$ is a finite inverse perfect semigroup of type 1 . Then $S$ is modular if and only if $S$ is one of M1, M2 and M3.

Proof. Sufficiency. Applying Lemma 4.3, we can show, by induction on the length of the chain, that M1 and M3 are modular. The result for M2 is well known.

Necessity. Let $S=\left(\cup_{i=1}^{n} S_{i}, \varphi_{i j}, i>j\right)$. By Theorem 1.11, $\left|S_{1}\right|<\left|S_{2}\right|$ $\leqslant \cdots \leqslant\left|S_{n}\right|$. Assume $n>2$ and $\left|S_{1}\right|=\cdots=\left|S_{l-1}\right|=1$ but $\left|S_{l}\right| \neq 1$ for some $l \leqslant n-2$, whence $\left|S_{n-1}\right| \neq 1,\left|S_{n}\right| \neq 1$. Let $I$ be the ideal of $S$ defined by $I=\cup_{i=1}^{n-2} S_{i}$. Then $S / I \cong\left(S_{n-1} \cup S_{n}\right)^{0}$. If $S$ is modular, then by Lemmas 1.4 
and 4.4, $S_{n-1} \cup S_{n}$ is modular. To prove necessity it is sufficient to prove the following:

(4.6) If $S$ is a perfect semigroup which is a semilattice of two non-trivial groups then $S$ is not modular.

Let $S=\left(S_{1} \cup S_{2}, \varphi_{i j}, i \geqslant j, i, j=1,2\right),\left|S_{1}\right|>1,\left|S_{2}\right|>1$. Let $\xi$ be the smallest semilattice congruence on $S$, namely, $\xi=\omega\left(S_{1}\right) \cup \omega\left(S_{2}\right)$. Let $\eta$ be the smallest group congruence on $S$ (see Corollary 3.4). In addition to $\xi$ and $\eta$, we define $\zeta$ by

$$
\zeta=\omega\left(S_{1}\right) \cup\left(\eta \mid S_{2}\right) .
$$

It is easy to see that $\zeta$ is a congruence. Since $\eta\left|S_{1}=\imath\left(S_{1}\right), \eta\right| S_{2} \subsetneq \omega\left(S_{2}\right)$, and hence $\zeta \subsetneq \xi$. We see

$$
\begin{aligned}
& \xi \vee \eta=\zeta \vee \eta=\omega(S), \\
& \xi \cap \eta=\zeta \cap \eta=\iota\left(S_{1}\right) \cup\left(\eta \mid S_{2}\right) .
\end{aligned}
$$

Therefore $S$ is not modular. Thus (4.6) has been proved.

Let $S$ be a finite inverse perfect semigroup of type 2. If $S$ is not a Brandt semigroup, $S=S_{0} \cup \bar{S}$, where $S_{0}=\mathscr{T}^{0}(m, G)$ is a Brandt semigroup and $\bar{S}=\left(\cup_{i=1}^{n} S_{i}, \varphi_{i j}, i \geqslant j\right)$ is of type 1. Assume $S$ is modular. Then $\bar{S}$ is modular by Lemma 4.4 , hence $\bar{S}$ is either M1, M2 or M3. Then we have

THEOREM 4.7. A finite inverse perfect semigroup $S$ of type 2 is modular if and only if $S$ is one of the following:

(M4) a Brandt semigroup;

(M5) a Brandt-group composition;

(M6) a Brandt-chain composition;

(M7) a Brandt-chain-group composition.

We call $S$ a Brandt-chain composition if $\bar{S}$ is M1; a Brandt-group composition if $\bar{S}$ is M2; a Brandt-chain-group composition if $\bar{S}$ is M3. Each $S$ is an ideal extension of a Brandt semigroup $S_{0}$ by $\bar{S}^{0}$ by means of permutations. In particular, $S$ in case of M6 or M7 is an ideal extension of $S_{0}$ by $\bar{S}^{0}$ by means of identity translations.

Proof. We want to show that all these are modular.

M4. Let $S=S_{0}=\mathscr{T}^{0}(m, G)$. By Lemma 1.9, non-universal congruences on $S_{0}$ are determined by congruences on $G$. So $L(S) \cong(L(G))^{1}$. By Lemma 4.1 $(L(G))^{1}$ is modular, and hence $S$ is modular. 
M5. Let $S=S_{0} \cup S_{1}$ where $S_{0}=\mathscr{N}^{0}(m, G)$ and $S_{1}$ is a group. We will prove that $L(S) \backslash\{\omega(S)\}$ is isomorphic to a sublattice of $L\left(S_{0}\right) \times L\left(S_{1}\right)$, whence $L(S)$ is modular. By Corollary 3.8 all congruences on $S$ are of the first kind except $\omega(S)$. By Theorem 3.7 if $\eta \in L(S)$ and $\eta \neq \omega(S)$, $\eta$ has the form $\eta=\rho_{0} \cup \rho_{1}$, where $\rho_{0} \in L\left(S_{0}\right), \rho_{1} \in L\left(S_{1}\right)$ and the pair satisfies condition (3.6). With a slight change of notation, let $\rho_{1}^{(0)}, \rho_{2}^{(0)} \in L\left(S_{0}\right), \rho_{1}^{(1)}, \rho_{2}^{(1)} \in L\left(S_{1}\right)$. Congruences on a group and a Brandt semigroup are permutable, that is, $\rho_{1}^{(0)} \cdot \rho_{2}^{(0)}=\rho_{2}^{(0)} \cdot \rho_{1}^{(0)}$ and $\rho_{1}^{(1)} \cdot \rho_{2}^{(1)}=\rho_{2}^{(1)} \cdot \rho_{1}^{(1)}$ as the product of relations. Hence $\rho_{1}^{(0)} \vee \rho_{2}^{(0)}=\rho_{1}^{(0)} \cdot \rho_{2}^{(0)}$, and $\rho_{1}^{(1)} \vee \rho_{2}^{(1)}=\rho_{1}^{(1)} \cdot \rho_{2}^{(1)}$. Then we can show that if pairs $\left(\rho_{1}^{(0)}, \rho_{1}^{(1)}\right)$ and $\left(\rho_{2}^{(0)}, \rho_{2}^{(1)}\right)$ satisfy condition (3.6), then the pairs $\left(\rho_{1}^{(0)} \cdot \rho_{2}^{(0)}, \rho_{1}^{(1)} \cdot \rho_{2}^{(1)}\right)$ and $\left(\rho_{1}^{(0)} \cap\right.$ $\left.\rho_{2}^{(0)}, \rho_{1}^{(1)} \cap \rho_{2}^{(1)}\right)$ satisfy (3.6). Note $\rho_{1}^{(0)} \cap \rho_{2}^{(0)} \neq \varnothing$ on $S_{0}$ and $\rho_{1}^{(1)} \cap \rho_{2}^{(1)} \neq \varnothing$ on $S_{1}$. Hence if $\rho_{1}^{(0)} \cup \rho_{1}^{(1)}, \rho_{2}^{(0)} \cup \rho_{2}^{(1)} \in L(S)$, then $\rho_{1}^{(0)} \cdot \rho_{2}^{(0)} \cup \rho_{1}^{(1)} \cdot \rho_{2}^{(1)},\left(\rho_{1}^{(0)} \cap \rho_{2}^{(0)}\right)$ $\cup\left(\rho_{1}^{(1)} \cap \rho_{2}^{(1)}\right) \in L(S)$. Immediately we have

$$
\begin{aligned}
& \left(\rho_{1}^{(0)} \cup \rho_{1}^{(1)}\right) \vee\left(\rho_{2}^{(0)} \cup \rho_{2}^{(1)}\right)=\left(\rho_{1}^{(0)} \vee \rho_{2}^{(0)}\right) \cup\left(\rho_{1}^{(1)} \vee \rho_{2}^{(1)}\right), \\
& \left(\rho_{1}^{(0)} \cup \rho_{1}^{(1)}\right) \cap\left(\rho_{2}^{(0)} \cup \rho_{2}^{(1)}\right)=\left(\rho_{1}^{(0)} \cap \rho_{2}^{(0)}\right) \cup\left(\rho_{1}^{(1)} \cap \rho_{2}^{(1)}\right) .
\end{aligned}
$$

Thus $L(S) \backslash\{\omega(S)\}$ is isomorphic to a sublattice of $L\left(S_{0}\right) \times L\left(S_{1}\right)$, and hence $L(S)$ is modular by Lemma 4.1.

M6 and M7. Let $S=S_{0} \cup \bar{S}, S_{0}=\mathscr{R}^{0}(m, G)$ and $\bar{S}=\cup_{i=1}^{n} S_{i}$, where $n>1, S_{i}=\left\{s_{i}\right\}$ for $1 \leqslant i<n$ and $S_{n}$ is a group (possibly trivial).

Let $L_{1}(S)$ and $L_{2}(S)$ denote the sets of congruences on $S$ of the first kind and second kind, respectively.

Because of identity composition, any pair $\left(\rho_{0}, \eta\right)$ of $\rho_{0} \in L\left(S_{0}\right)$ and $\eta \in L(\bar{S})$ satisfies condition (3.6). Therefore we have $L_{1}(S) \cong L\left(S_{0}\right) \times L(\bar{S})$.

As $S_{1}=\left\{s_{1}\right\}$, every congruence $\eta$ on $\bar{S}$ has the property that $\bar{S} / \eta$ has zero, and hence a congruence $\eta_{2}$ on $S$ of the second kind can be obtained from every congruence $\eta$ on $\bar{S}$. It is easily shown that $L_{2}(S) \cong L(\bar{S})$. Then we have

$$
\begin{aligned}
& L_{1}(S) \cong\left\{(\xi, \eta): \xi \in L\left(S_{0}\right), \eta \in L(\bar{S})\right\} \quad \text { under } \xi \cup \eta \mapsto(\xi, \eta), \\
& L_{2}(S) \cong\{(1, \eta): \eta \in L(\bar{S})\} \quad \text { under } \zeta \mapsto(1, \zeta \mid \bar{S}),
\end{aligned}
$$

and also

$$
\begin{aligned}
& \left(\xi_{1}, \eta_{1}\right) \vee\left(1, \eta_{2}\right)=\left(1, \eta_{1} \vee \eta_{2}\right), \\
& \left(\xi_{1}, \eta_{1}\right) \wedge\left(1, \eta_{2}\right)=\left(\xi_{1}, \eta_{1} \wedge \eta_{2}\right)
\end{aligned}
$$

Therefore we have

$$
L(S)=L_{1}(S) \cup L_{2}(S) \simeq\left(L\left(S_{0}\right)\right)^{1} \times L(\bar{S}) .
$$

Since $L\left(S_{0}\right)$ and $L(\bar{S})$ are modular, $L(S)$ is modular. This completes the proof of Theorem 4.7. 
In summary

THEOREM 4.8. Let $S$ be a finite inverse perfect semigroup. Then $S$ is modular if and only if $S$ does not contain more than one non-trivial group component in the greatest semilattice decomposition of $S$; equivalently, $S$ is one of the following:

(M1) a chain;

(M2) a group;

(M3) a chain-group composition;

(M4) a Brandt semigroup;

(M5) a Brandt-group composition;

(M6) a Brandt-chain composition;

(M7) a Brandt-chain-group composition.

Remark on Modularity of M1. Without assuming finiteness, the lattice of congruences on any chain is modular [distributive]. A congruence on a chain as a semilattice is its partition into non-overlapping segments by Lemma 3.1, and its partition gives also a congruence on the chain as a lattice (Exercise 1, page 138, Birkhoff (1967)). By Funayama and Nakayama's theorem (Theorem 9, page 138, Birkhoff (1967)), the lattice of congruences on any lattice is complete Browerian, but a Browerian lattice is distributive by Theorem 18, page 45 , Birkhoff (1967). Incidentally we have that the lattice of congruences on a lattice $S(\vee, \wedge)$ equals the lattice of congruences on the join [meet] semilattice $S(\vee)$ $[S(\wedge)]$ if and only if $S(\vee, \wedge)$ is a chain.

ACKNOWLEDGEMENT. The authors express their heartfelt thanks to the referee for his kind suggestion.

\section{References}

G. Birkhoff (1967), Lattice theory (Colloq. Publ. 25, Amer. Math. Soc., Providence, R.I).

A. H. Clifford and G. B. Preston (1961), The algebraic theory of semigroups, Vol. 1 (Math. Surveys, Amer. Math. Soc., Providence, R.I).

A. H. Clifford and G. B. Preston (1967), The algebraic theory of semigroups, Vol. 2 (Math. Surveys, Amer. Math. Soc., Providence, R.I).

V. A. Fortunatov (1970), 'Perfect semigroups decomposable in a semi-lattice of rectangular groups'. Studies in algebra 2, 67-78 (Saratov Univ. Press (in Russian)).

V. A. Fortunatov (1972), 'Perfect semigroups', Izv. Vyss. Učebn. Zaved. Matematika 3, 80-90 (in Russian).

V. A. Fortunatov (1974), 'Varieties of perfect algebras', Studies in algebra 4, 110-114 (Saratov Univ. Press (in Russian)). 
V. A. Fortunatov (1977), 'Congruences on simple extensions of semigroups', Semigroup Forum 13, 283-295.

J. M. Howie (1976), An introduction to semigroup theory (Academic Press).

M. Petrich (1973), Introduction to semigroups (Merrill Publ. Co., Columbus, Ohio).

N. R. Reilly and H. E. Scheiblich (1967), 'Congruences on regular semigroups', Pacific J. Math. 23, 349-360.

T. Tamura (1956), 'Theory of construction of finite semigroups I', Osaka J. Math. 8, 243-261.

T. Tamura (1960), 'Decompositions of a completely simple semigroup', Osaka J. Math. 12, 269-275.

V. V. Vagner (1968), 'Algebraic topics of the general theory of partial connections in fiber bundles', Izv. Vyss. Učebn. Zaved. Matematika 11, $26-32$.

R. J. Wielandt (1964), Finite permutation groups (Academic Press).

Department of Mathematics and Statistics

California State University at Sacramento Sacramento, California 95819

U.S.A.
Department of Mathematics University of California at Davis Davis, California 95616

U.S.A. 